LBL-37198

UCB-PTH-95/13

LPTHE-Orsay 95/38

June 1995

hep-th/9506207

\title{
S-DUALITY CONSTRAINTS ON EFFECTIVE POTENTIALS FOR GAUGINO CONDENSATION⿴囗十
}

\author{
Pierre Binétruy \\ Laboratoire de Physique Théorique et Hautes Energies, П] \\ Université Paris-Sud, F-91405 Orsay, France \\ and \\ Mary K. Gaillard \\ Department of Physics and Theoretical Physics Group, Lawrence Berkeley \\ Laboratory, University of California, Berkeley, California 94720
}

\begin{abstract}
We clarify the role of approximate $S$-duality in effective supergravity theories that are the low energy limits of string theories, and show how this partial symmetry may be used to constrain effective lagrangians for gaugino condensation.
\end{abstract}

${ }^{*}$ This work was supported in part by the Director, Office of High Energy and Nuclear Physics, Division of High Energy Physics of the U.S. Department of Energy under contract DE-AC03-76SF00098, in part by the National Science Foundation under grant PHY-9021139

$\dagger$ Laboratoire associé au CNRS-URA-D0063. 


\section{Introduction}

It has long been understood that abelian gauge fields can be coupled to scalar fields in such a way that the equations of motion are invariant under a group of "duality" transformations [1] that interchange the gauge field strength $F_{\mu \nu}$ with its dual $\tilde{F}_{\mu \nu}$, provided the (noncanonical) kinetic energy term of the scalar field(s) [hereafter referred to as the dilaton(s)] that couple to the gauge fields satisfies certain constraints. This is an automatic feature of ungauged extended supergravity theories [2]. A special example of this class of models is the dilaton plus Yang-Mills sector of effective $N=1$ supergravity theories obtained from superstrings in the limit where the gauge and Yukawa couplings are set to zero. In this case the group of duality transformations is $S L(2, \mathcal{R})$, which has as a discrete subgroup $S L(2, \mathcal{Z})$ that includes the transformation $S \rightarrow 1 / S$, often referred to as $S$-duality. This is analogous to the modular group, known to be exact to all orders of string perturbation theory [3], which contains a subgroup $S L(2, \mathcal{Z})$ that includes the transformation $T \rightarrow 1 / T$, where $T$ is a modulus chiral supermultiplet. The vev of its scalar component $t$ determines the radius of compactification. In models from orbifold compactification, this discrete symmetry is generally a subgroup of a classical continuous symmetry that contains $S L(2, \mathcal{R})$ as a subgroup. Such symmetries are anomalous at the quantum level of the effective field theory since, for example, in supergravity they entail chiral transformations on fermions. In the case of modular invariance counterterms [4, 5] must be added to the effective field theory so as to restore the discrete modular symmetry.

It has been conjectured [6] that a similar situation might hold with respect to $S$-duality. Since the vev of the complex scalar field $s$ that is the scalar member of the dilaton supermultiplet $S$ determines the gauge coupling constant: $\langle s\rangle=g^{-2}-i \theta / 8 \pi^{2}$, this corresponds to strong/weak coupling duality. Recently there has been considerable interest in $S$-duality from both the string [7, 8] and field [7, 9, 10] theory points of view. In particular, it has 
been shown that the $S L(2, \mathcal{Z})$ subgroup of $S L(2, \mathcal{R})$ that is generated by the elements $\theta \rightarrow \theta+2 \pi$ and $4 \pi s \rightarrow 1 / 4 \pi s$ relates different string theories [B], and also that certain theories are $S$-duality invariant [9] under transformations involving both elementary fields and nonperturbative solutions.

In this paper we restrict our attention to effective field theories of the type that have been obtained explicitly in orbifold compactifications. In these theories $S$-duality, which we define hereafter as the $S L(2, \mathcal{R})$ group of duality transformations among elementary fields, is a symmetry of the equations of motion only of the dilaton-gauge-gravity sector in the limit of vanishing gauge couplings - that is, the limit in which the gauge group reduces to $U(1)^{n}$, where $n$ is the total number of gauge degrees of freedom. Since the effective superpotential [11, 12] that parameterizes gaugino condensation is induced by the nonabelian self-couplings of Yang-Mills fields and by their gauge couplings to chiral supermultiplets, this term must explicitly break duality. Nevertheless, approximate $S$-duality may be a useful tool in parameterizing additional quantum effects that arise from the couplings of the Yang-Mills sector to the gravity and dilaton sectors. In Section 2 we show how the dilaton couplings in the chiral multiplet formulation are related to the general formulation [1] of duality invariance for interacting vector fields, and use this formulation to obtain the duality transformation law for the composite chiral multiplet that is interpreted as the lightest bound state of a confined Yang-Mills sector. In section 3 we give examples of how this transformation property may be used to constrain effective potentials in the chiral formulation. We will consider both the chiral multiplet and the linear multiplet formulations for the dilaton superfield.

\section{Duality transformations}

We first recall the relevant elements of the general formalism constructed in [四] for a noncompact group $\mathcal{G} \subset S p(2 n, \mathcal{R})$ of duality transformations on 
$n$ vector field strengths $F$. The scalars are valued in the coset space $\mathcal{G} / \mathcal{K}$, where $\mathcal{K} \subset U(n)$ is the maximal compact subgroup of $\mathcal{G}$, and they can be represented by a group element $g$ :

$$
g=\left(\begin{array}{cc}
\phi_{0} & \phi_{1}^{*} \\
\phi_{1} & \phi_{0}^{*}
\end{array}\right), \quad \phi_{0}^{\dagger} \phi_{0}-\phi_{1}^{\dagger} \phi_{1}=1
$$

that is restricted to the special form:

$$
g=\exp \left(\begin{array}{cc}
0 & P^{*} \\
P & 0
\end{array}\right) .
$$

Under $\mathcal{G}$ the vector field strengths and scalar fields, respectively, transform as

$$
\begin{gathered}
\left(\begin{array}{c}
F+i G \\
F-i G
\end{array}\right) \rightarrow g_{0}\left(\begin{array}{c}
F+i G \\
F-i G
\end{array}\right), \quad g_{0}=\exp \left(\begin{array}{cc}
T & V^{*} \\
V & T^{*}
\end{array}\right), \\
g \rightarrow g_{0} g k^{-1}=\exp \left(\begin{array}{cc}
0 & P^{* *} \\
P^{\prime} & 0
\end{array}\right)
\end{gathered}
$$

where $\tilde{G}^{\mu \nu}=\frac{1}{2} \epsilon^{\mu \nu \rho \sigma} G_{\rho \sigma}=2 \partial \mathcal{L} / \partial F^{\mu \nu}, T=-T^{\dagger}$ is a compact generator of $\mathcal{K}$, $V=V^{T}$ is a noncompact generator of $\mathcal{G} / \mathcal{K}$, and $k=k\left(g_{0}, P\right)$ is an element of $\mathcal{K}$ with field-dependent parameters chosen so as to preserve the off-diagonal form of $\ln g$. The symmetry of $P$ implies $\phi_{0}^{\dagger}=\phi_{0}, \phi_{1}^{T}=\phi_{1}$. The lagrangian describing these bose degrees of freedom, is

$$
\begin{aligned}
\mathcal{L} & =-\frac{1}{4} F_{\mu \nu} \operatorname{Re} f F^{\mu \nu}-\frac{1}{4} F_{\mu \nu} \operatorname{Im} f \tilde{F}^{\mu \nu}+\frac{1}{2} \operatorname{Tr}\left(P^{\mu} P_{\mu}\right), \quad f=\frac{1}{2}\left(\frac{1-Z^{*}}{1+Z^{*}}\right), \\
Z & =Z^{T}=\phi_{1}\left(\phi_{0}\right)^{-1}, \quad P_{\mu}=g^{-1} D_{\mu} g \equiv g^{-1} \partial_{\mu} g-Q_{\mu},
\end{aligned}
$$

where $Q_{\mu}$ and $P_{\mu}$ are the parts in the Lie algebra of $\mathcal{K}$ and of $\mathcal{G} / \mathcal{K}$, respectively, of the the element $g^{-1} \partial_{\mu} g=Q_{\mu}+P_{\mu}$ of the Lie algebra of $\mathcal{G}$. The equations of motion derived from the lagrangian (2.4) are invariant under the

\footnotetext{
${ }^{1}$ The normalization of the vector kinetic term here differs by a factor two from that in [1], where it was chosen to coincide with the canonical one in the limit $Z \rightarrow 0$.
} 
transformations (2.3), although the action is not.7 Couplings to other fields $\psi_{i}$ are determined by their transformation properties under $\mathcal{K}$; the equations of motion of the following lagrangian are invariant under (2.3) with $\psi_{i} \rightarrow k_{i}\left(g_{0}, P\right) \psi_{i}$, where $k_{i}$ represents $\mathcal{K}$ on the multiplet of fields $\psi_{i}$ :

$$
\mathcal{L}(\psi)=\bar{\psi} \gamma^{\mu} D_{\mu} \psi+\alpha^{i j} \bar{\psi}_{i} \gamma^{\mu} P_{\mu} \psi_{j}+\cdots, \quad D_{\mu} \psi=\partial_{\mu}+Q_{\mu} \psi,
$$

where the $\alpha^{i j}$ are constants, and the dots represent possible $\mathcal{G}$-invariant operators of higher dimension, as well as operators constructed from the field strength $F_{\mu \nu}$, an arbitrary antisymmetric tensor $H_{\mu \nu}(\psi)$, and their duals [1] .

Here we are interested in the simplest realization of the above construction, namely $\mathcal{G}=S L(2, \mathcal{R}), \mathcal{K}=U(1)$. Then the $n \times n$ matrices $\phi_{i}, T, V, Z, P$ and $f$ are proportional to the unit matrix. The dilaton $s$ from superstring theory is identified as

$$
s=\frac{1}{2}\left(\frac{1-Z^{*}}{1+Z^{*}}\right)=f .
$$

Expressing $\phi_{i}$ in terms of $s$ we obtain:

$$
\begin{aligned}
P_{\mu} & =-\frac{1}{s+\bar{s}}\left(\begin{array}{cc}
0 & \left(\eta^{*}\right)^{2} \partial_{\mu} \bar{s} \\
\eta^{2} \partial_{\mu} s & 0
\end{array}\right), \quad \frac{1}{2} \operatorname{Tr}\left(P_{\mu} P^{\mu}\right)=\frac{\partial_{\mu} s \partial^{\mu} \bar{s}}{s+\bar{s}} \\
\eta & =\left(\eta^{*}\right)^{-1}=\sqrt{\frac{1+2 \bar{s}}{1+2 s}}
\end{aligned}
$$

Inserting (2.6) and (2.7) in (2.4), we recover the standard lagrangian for the string dilaton with Kähler potential $K(S, \bar{S})=-\ln (S+\bar{S})$. In addition we have

$$
Q_{\mu}=\frac{1}{2(s+\bar{s})}\left(\begin{array}{cc}
\partial_{\mu} s-\partial_{\mu} \bar{s} & 0 \\
0 & \partial_{\mu} \bar{s}-\partial_{\mu} s
\end{array}\right)+\left(\begin{array}{cc}
\eta^{*} \partial_{\mu} \eta & 0 \\
0 & \eta \partial_{\mu} \eta^{*}
\end{array}\right) .
$$

The action of $S L(2, \mathcal{R})$ on $s$ takes the usual form

$$
s \rightarrow s^{\prime}=\frac{a s-i b}{i c s+d}, \quad a, b, c, d \in \mathcal{R}, \quad a d-b c=1 .
$$

\footnotetext{
${ }^{2}$ An invariant action for supergravity with an abelian gauge group has been constructed in [7] by sacrificing manifest general coordinate invariance.
} 
This corresponds in (2.3) to

$$
\begin{aligned}
g_{0} & =\left(\begin{array}{cc}
p & q^{*} \\
q & p^{*}
\end{array}\right), \quad k=\left(\begin{array}{cc}
\zeta & 0 \\
0 & \zeta^{*}
\end{array}\right), \\
p & =\frac{1}{2}(a+d)+\frac{1}{4} c-i b, \quad q=\frac{1}{2}(d-a)+\frac{1}{4} c+i b, \\
\zeta & =\left(\zeta^{*}\right)^{-1}=\frac{\eta}{\eta^{\prime}} \xi^{*}, \quad \eta^{\prime}=\eta\left(s^{\prime}\right), \quad \xi=\sqrt{\frac{-i c \bar{s}+d}{i c s+d} .}
\end{aligned}
$$

A Weyl fermion $\psi_{n}$ of $U(1)$ charge $n$ transforms as

$$
\psi_{n} \rightarrow \psi_{n}^{\prime}=\zeta^{n} \psi_{n},
$$

and its $S L(2, \mathcal{R})$ invariant kinetic energy is given by

$$
\begin{array}{r}
\mathcal{L}_{\text {K.E. }}\left(\psi_{n}\right)=\bar{\psi}_{n} \gamma^{\mu} D_{\mu} \psi_{n}=\bar{\psi}_{n} \gamma^{\mu}\left(\partial_{\mu}+\frac{i n}{s+\bar{s}} \partial_{\mu} \operatorname{Im} s+n \eta^{n} \partial_{\mu} \eta\right) \psi_{n} \\
=\frac{i}{(s+\bar{s})^{2 \beta}} \bar{f}_{n, \beta} \gamma^{\mu}\left(\partial_{\mu}-\frac{2 \beta}{s+\bar{s}} \partial_{\mu} \operatorname{Re} s+\frac{i n}{s+\bar{s}} \partial_{\mu} \operatorname{Im} s\right) f_{n, \beta},(2.2)
\end{array}
$$

where we have defined $f_{n, \beta}=\eta^{n}(s+\bar{s})^{\beta} \psi_{n}$; under $S L(2, \mathcal{R})$ :

$$
f_{n, \beta} \rightarrow f_{n, \beta}^{\prime}=\xi^{-n}|i s c+d|^{-2 \beta} f_{n, \beta}=\xi^{-2 \beta-n}(i c s+d)^{-2 \beta} f_{n, \beta} .
$$

In a supersymmetric theory the transformation property (2.9) of $s$ applies to the dilaton chiral supermultiplet $S(\theta)=s+\theta \chi_{S}+\cdots$ :

$$
S(\theta) \rightarrow S^{\prime}(\theta)=\frac{a S\left(\theta^{\prime}\right)-i b}{i c S\left(\theta^{\prime}\right)+d} .
$$

This effects a Kähler transformation:

$$
K \rightarrow K+F+\bar{F}, \quad F=\ln (i c s+d), \quad \theta^{\prime}=e^{\frac{1}{4}(F-\bar{F})} \theta=\xi^{-\frac{1}{2}} \theta,
$$

from which one can derive the transformation properties of the component fields of $S$; in particular

$$
\chi_{S} \rightarrow \chi_{S}^{\prime}=\xi^{-\frac{1}{2}}(i c s+d)^{-2} \chi_{S} .
$$


Thus we can identify $\chi_{S} \equiv f_{-\frac{3}{2}, 1}$ in (2.13). It is straightforward to verify that $\mathcal{L}\left(f_{-\frac{3}{2}, 1}\right)$ given in $(2.12)$ coincides with the kinetic energy term for $\chi_{S}$ in the standard supergravity lagrangian [13, 14].

The other fermion fields that couple to the dilaton are the gauginos $\lambda$ with kinetic energy term:

$$
\mathcal{L}_{\text {K.E. }}(\lambda)=\frac{1}{2} \bar{\lambda}_{L}\left(\not \partial+\frac{i}{2} \frac{\not \operatorname{Im} s}{s+\bar{s}}\right) \tilde{\lambda}_{L}+\text { h.c., } \quad \tilde{\lambda}=\sqrt{\operatorname{Re} s} \lambda .
$$

Comparing (2.17) with (2.12), the (canonically normalized) field $\tilde{\lambda}_{L}$ is identified as $\tilde{\lambda}_{L} \equiv f_{\frac{1}{2}, 0}$, from which it follows that $\lambda_{L} \equiv f_{\frac{1}{2},-\frac{1}{2}}$, with its transformation law given by (2.13) as:

$$
\lambda_{L} \rightarrow \lambda_{L}^{\prime}=\xi^{\frac{1}{2}}(i c s+d) \lambda_{L} .
$$

This immediately gives the transformation law for the gauge field strength supermultiplet $W_{\alpha}$, where $\alpha$ is a Dirac index:

$$
W_{\alpha}(\theta) \rightarrow W_{\alpha}^{\prime}(\theta)=\xi^{\frac{1}{2}}(i c s+d) W_{\alpha}\left(\theta^{\prime}\right) .
$$

Having established the transformation laws for the superfields $S$ and $W_{\alpha}$, and using the invariance under $S L(2, \mathcal{R})$ of the supervielbein, one can directly check that the supergravity equations of motion [14] are invariant under $S L(2, \mathcal{R})$ when the the theory is limited to this set of fields. In this case they reduce to:

$$
\begin{aligned}
& R=R^{\dagger}=0, \\
& G_{b}+\frac{1}{8(s+\bar{s})^{2}} \bar{\sigma}_{b}^{\dot{\alpha} \alpha} \mathcal{D}_{\alpha} S \mathcal{D}_{\dot{\alpha}} \bar{S}-\frac{(S+\bar{S})}{8} \bar{\sigma}_{b}^{\dot{\alpha} \alpha} W_{\alpha} W_{\dot{\alpha}}=0, \\
& \frac{S}{2} \mathcal{D}^{\alpha} W_{\alpha}-\frac{1}{2} \mathcal{D}_{\alpha} S W^{\alpha}+\text { h.c. }=0, \\
& (S+\bar{S})^{-2} \bar{F}^{S}+\frac{1}{4} W^{\alpha} W_{\alpha}=0,
\end{aligned}
$$

where $b$ is a Lorentz index, $F^{S}=-\frac{1}{4} \mathcal{D}^{\alpha} \mathcal{D}_{\alpha} S$, and the superfields $R$ and $G_{b}$ describe the supergravity multiplet; they are related to the curvature 
superfield by:

$$
R_{b a}^{\dot{\delta} \dot{\gamma}}=8\left(\bar{\sigma}_{b a} \epsilon\right)^{\dot{\delta} \dot{\gamma}} R, \quad R_{\delta b a}^{\dot{\gamma}}=-2 i G^{d}\left(\sigma^{c} \epsilon\right)_{\delta}^{\dot{\gamma}} \epsilon_{d c b a} .
$$

Using the Bianchi identity:

$$
\mathcal{D}^{\alpha} W_{\alpha}=\mathcal{D}_{\dot{\alpha}} W^{\dot{\alpha}}
$$

it is straightforward to show that these equations are invariant under the $S L(2, \mathcal{R})$ transformations defined by $(2.14)$ and $(2.19)$. It follows from the latter that the composite superfield

$$
U \sim W^{\alpha} W_{\alpha}
$$

used in effective supergravity lagrangians for gaugino condensation transforms as

$$
U(\theta) \rightarrow U^{\prime}(\theta)=\xi(i c s+d)^{2} U(\theta) .
$$

As shown in [15], since the Kähler weight of the chiral superfield $W_{\alpha}$ differs from that of an ordinary chiral superfield such as $S$, the composite field $U$, in the context of supergravity, is related to an ordinary chiral field $H$ by:

$$
U=e^{K / 2} H^{3}
$$

where $K$ is the full Kähler potential; classically:

$$
K=-\ln (S+\bar{S})+G(Z, \bar{Z}), \quad U=\frac{e^{G / 2} H^{3}}{(S+\bar{S})^{\frac{1}{2}}},
$$

where $Z$ represents chiral multiplets other than the dilaton. Since under (2.14) $(S+\bar{S}) \rightarrow|i c S+d|^{-2}(S+\bar{S})$, we obtain the transformation property

$$
H(\theta) \rightarrow H^{\prime}(\theta)=(i c S+d)^{\frac{1}{3}} H(\theta)
$$

We emphasize that, although the equations of motions (2.20) make no explicit reference to the nonabelian nature of the gauge field strength, they 


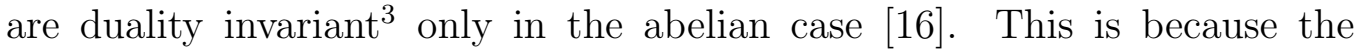
covariant spinorial derivatives (or, in the case of nonsupersymmetric theories, the covariant derivatives) have an implicit dependence on the gauge potential. Furthermore, even in the abelian case duality is broken if gauge couplings to matter are introduced, since this also involves the gauge potential explicitly. Finally, $S$-duality is broken by the presence of a superpotential $W(Z)$ through the appearance of the noninvariant factor $e^{K(s, \bar{s})}$ in the corresponding scalar potential and Yukawa couplings.

Finally we note that while the equations of motion $(2.20)$ are $S L(2, \mathcal{R})$ invariant, the Yang-Mills superfield lagrangian [14] is not':

$\mathcal{L}_{Y M}=\frac{1}{8} \int d^{4} \theta \frac{E}{R} S W^{\alpha} W_{\alpha}+$ h.c.,$\quad S W^{\alpha} W^{\alpha} \rightarrow|i c S+d|\left(a-\frac{i b}{S}\right) S W^{\alpha} W^{\alpha}$.

This result will have implications for attempts to impose approximate $S L(2, \mathcal{R})$ invariance on effective lagrangians for gaugino condensation, to be discussed in the next section.

\section{Gaugino condensation}

\section{A. Chiral multiplet formulation}

The superpotential for gaugino condensation was first derived by Veneziano and Yankielowicz [11] in the context of a supersymmetric renormalizable Yang-Mills theory, by imposing the correct chiral and conformal anomalies. Their result was extended to include the dilaton by Taylor [12. In the superfield formulation [14] of supergravity this leads to the potential term [15],

\footnotetext{
${ }^{3}$ There is evidence [9] for invariance under duality transformations involving soliton solutions in $N=2$ supersymmetric Yang-Mills theories with vanishing $\beta$-functions (which includes the case $N=4$ ).

${ }^{4}$ See however [7].
} 
expressed in terms of the chiral multiplet $H$ introduced in $(2.24)$, of the form

$$
\begin{aligned}
\mathcal{L}_{\text {pot }} & =\mathcal{L}_{C}+\mathcal{L}_{Q}=\frac{1}{2} \int d^{4} \theta \frac{E}{R} e^{K / 2}\left(W_{C}+W_{Q}\right), \\
W_{C} & =\frac{1}{4} S H^{3}, \quad W_{Q}=\frac{b_{0}}{2} H^{3} \ln (H / \mu)
\end{aligned}
$$

with $b_{0}$ the group theory constant that determines the appropriate $\beta$-function. $\mathcal{L}_{C}$ and $\mathcal{L}_{Q}$ are usually interpreted as the classical and quantum contributions, respectively, to the effective potential for gaugino condensation. As anticipated in the introduction, $\mathcal{L}_{Q}$ is not $S L(2, \mathcal{R})$ invariant. However, neither is $\mathcal{L}_{C}$, since, by construction, it has the same transformation property as (2.27). In the general formulation [1] of duality transformations, couplings of the dilaton(s) to matter entail duality invariance of the corresponding terms in the lagrangian, as opposed to their couplings to gauge fields, which are only an invariance of the equations of motion. This mismatch may be traced to the fact that we used the Bianchi identity (2.21) to obtain the invariance of the equations of motion for the underlying theory expressed in terms of the Yang-Mills field strength $W_{\alpha}$. The identification (2.22), together with the constraint

$$
\left(\mathcal{D}^{\alpha} \mathcal{D}_{\alpha}-24 R^{\dagger}\right) W^{\beta} W_{\beta}-\left(\mathcal{D}_{\dot{\alpha}} \mathcal{D}^{\dot{\alpha}}-24 R\right) W_{\dot{\beta}} W^{\dot{\beta}}=\text { total derivative }
$$

implies a constraint on the superfield $H$ that is not satisfied for an ordinary superfield. This suggests a possible inconsistency in all chiral multiplet formulations of gaugino condensation [11, 12, 15, 17, 18], especially those treatments 19 à la Nambu-Jona-Lasinio in which the use of a Lagrange multiplier imposes the operator identity $U=W^{\alpha} W_{\alpha}$. We will return to this point in section 3.B below.

In this paper we consider a toy model with a single modulus superfield $T$, and set gauge nonsinglet matter fields to zero. These simplifications do not affect the generality of our results. In 15] we showed that the (continuous) modular invariance of the classical supergravity theories requires that the 
Kähler potential depend on the composite field $H$ only through the invariant $|H|^{2} /(T+\bar{T})$, and we adopted the no-scale form

$$
\begin{aligned}
K & =-\ln (S+\bar{S})-3 \ln (T+\bar{T})-3 \ln \left(1-f(S, \bar{S}) \frac{|H|^{2}}{T+\bar{T}}\right) \\
& =-\ln (S+\bar{S})-3 \ln \left(T+\bar{T}-f(S, \bar{S})|H|^{2}\right)
\end{aligned}
$$

The effective theory constructed by combining (3.3) with (3.1) was subsequently modified [17, 18 by including an additional $T$-dependence through the Dedekind function $\eta(T)$ in such a way as to restore invariance under the discrete modular group. Although this modification was regarded as a parameterization of threshold corrections [ [ heavy string modes in some orbifold compactifications, none of the above models is truly consistent with string theory, since they do not incorporate the Green-Schwarz counterterm [5] that cancels part (or in many cases all [20]) of the modular anomaly that arises from perturbative field theory quantum corrections. The effect of the Green-Schwarz term is to modify the Kähler potential by

$$
\ln (S+\bar{S}) \rightarrow \ln (S+\bar{S}-b G), \quad G=-3 \ln (T+\bar{T})
$$

where $b=2 b_{0} / 3$, and $b_{0}=.56$ is the constant that determines the $E_{8} \beta$ function. This modification spoils the no-scale feature of the Kähler potential and generally leads to an unbounded potential.

In this section we consider a prototype model in which the hidden gauge group is $E_{8}$, in which case the anomaly is completely cancelled by the GreenSchwarz term, and furthermore there is no ambiguity in constructing an effective composite potential. That is, since the modular transformation laws are now, with $\alpha, \beta, \gamma, \delta \ni \mathcal{R}, \alpha \delta-\beta \gamma=1$ :

$$
\begin{aligned}
& T \rightarrow T^{\prime}=\frac{\alpha T-i \beta}{i \gamma T+\delta}, \quad G \rightarrow G+F+\bar{F}, \quad F=3 \ln (i \gamma T+\delta) \\
& H \rightarrow H^{\prime}=e^{-F / 3} H, \quad S \rightarrow S^{\prime}=S+b F,
\end{aligned}
$$


the structure (3.1), which can be understood [21] as arising from coupling constant renormalization, coincides with the requirements of modular invariance.

We study the effective lagrangian for the composite superfield $H$ under the assumptions that modular invariance is exact to all orders, and that $S$ duality, as defined by (2.14) and (2.26), with $T \rightarrow T^{\prime}=T$, is recovered to leading order in $g^{2} \equiv(\operatorname{Re} s)^{-1} \equiv \sigma$. $S$-duality determines the function $f(S, \bar{S})$ in $(3.3): f(S, \bar{S})=(S+\bar{S})^{\frac{1}{3}}$; we therefore adopt the Kähler potential:

$$
\begin{aligned}
& K=-\ln M-3 \ln \left(1-\left(M^{\prime}\right)^{\frac{1}{3}} Q\right)+G, \quad L^{-1}=S+\bar{S}-b G, \\
& M=L^{-1}+3 b_{1} \ln Q, \quad M^{\prime}=L^{-1}+3 b_{2} \ln Q, \quad Q=|H|^{2} e^{G / 3} .
\end{aligned}
$$

The rationale is as follows. Modular invariance requires $K=G+k(L, Q)$. In the limit $g^{2} \rightarrow 0$, i.e., $\sigma \rightarrow \infty$, (3.6) is $S$-duality invariant and reduces to the form (3.3). By matching string one-loop calculations with field theory ones, it was shown in [22] that at the string scale the Kähler potential is precisely (3.6) with $b_{1}=b_{2}=H=0$; the modular invariant scalar field

$$
\ell=[s+\bar{s}+3 b \ln (t+\bar{t})]^{-1}=2 g^{2}
$$

is twice the squared gauge coupling constant at that scale [22, 23]. However, we are interested in the effective theory at the condensation scale; one possibility is that we should replace everywhere the string scale coupling constant by the running coupling constant evaluated at the condensation scale, which would correspond to $b_{1}=b_{2}=b$. In this case the theory is of the no-scale form, and the potential is positive semi-definite.

For the superpotential we take the most general form consistent with modular invariance of string perturbation theory:

$$
W=c+W_{0}, \quad W_{0}=e^{-S / b} F(Y), \quad Y=H e^{S / 3 b}
$$

\footnotetext{
${ }^{5}$ We use reduced Planck mass units: $8 \pi G_{N}=1 / m_{P l}^{2}=1$, and throughout this subsection we use upper case letters for chiral scalar superfields and lower case for the corresponding complex scalars.
} 
that is, we take $W_{0}(S, H)$ to be modular invariant but allow for a constant term that breaks modular invariance, that might arise [24] from a classical nonperturbative effect such as the vev of the three-form $H_{\ell m n}$ of ten dimensional supergravity. In the standard formulation [11, 12, 15, 17, 21, 18] of gaugino condensation $F(Y) \propto Y^{3} \ln Y$. However, as noted above this is not invariant under $S$-duality in the limit $g^{2} \rightarrow 0(\sigma \rightarrow \infty)$. If we adopt the point of view that $S$-duality should be recovered in this limit, we require

$$
\lim _{y \rightarrow \infty} F(y) \sim y^{n}(\ln y)^{m}, \quad n<3 .
$$

Such a superpotential could be interpreted as arising from purely nonperturbative effects, with, for $b_{2}=b$, the Yang-Mills wave function renormalization encoded in the Kähler potential, rather than the superpotential - in contrast to (3.1). A similar reinterpretation was used in 18 to recover a positive semi-definite no-scale potential in the presence of $\eta(T)$-dependent terms.

We have studied the potential for three choices of $b_{1}, b_{2}$ in (3.6) that we enumerate below. An interesting question that we address is whether or not it is possible to generate a bounded potential (more precisely, one with vanishing vacuum energy) with supersymmetry breaking without the introduction of a constant $c$ in the superpotential. The Kähler potentials we consider are by no means the most general. For example, one-loop corrections [25] induce a term:

$$
\mathcal{L}+\mathcal{L}_{1-\text { loop }} \ni-3 \int d^{4} \theta E+\frac{N_{G}}{128 \pi^{2}} \int d^{4} \theta E(S+\bar{S})^{2}\left|W^{\alpha} W_{\alpha}\right|^{2} \ln \Lambda^{2}
$$

where $\Lambda$ is the effective cut-off, that we take here to be a constant, and $N_{G}$ is the number of gauge degrees of freedom. Since this term arises only from couplings of the gauge sector to the dilaton sector it is $S$-duality invariant, and would by itself generate a kinetic term for the composite field $H$; making the replacement $W^{\alpha} W_{\alpha} \rightarrow U$, the classical Kähler potential is modified as

$$
\begin{aligned}
e^{-K / 3} & \rightarrow e^{-K / 3}\left(1-\frac{\alpha}{3} e^{K}(S+\bar{S})^{2}|H|^{6}\right), \quad \alpha=\frac{N_{G} \ln \Lambda^{2}}{128 \pi^{2}} \\
K & \rightarrow K-3 \ln \left(1-\frac{\alpha}{3} \frac{S+\bar{S}}{(T+\bar{T})^{3}}|H|^{6}\right)+O\left(\hbar^{2}\right),
\end{aligned}
$$


where the $O\left(\hbar^{2}\right)$ terms include the substitution $S+\bar{S} \rightarrow L^{-1}$ when exact modular invariance is imposed. Similar higher dimensional operators arise [26] from string corrections even at the classical level; it remains to be seen whether or not such a Kähler potential allows for a viable effective potential for the composite multiplet. Since the models we consider possess a continuous modular symmetry, the vev $\langle t\rangle$, that fixes the radius of compactification, is undetermined. This degeneracy of the vacuum could be lifted by quantum corrections in the effective field theory and/or by string corrections such as $\eta(T)$-dependent threshold corrections.

\section{I. $b_{1}=b_{2}=0$.}

This corresponds to using only the string coupling constant in the Kähler potential. We considered a parameterization of the superpotential $F(y)=$ $\sum_{n} a_{n} y^{n}$. The requirement that the potential be positive semi-definite constrains the values of $n$ : $n>.4238$ or $n<-4.74$ (and thus $c=0$ ). We studied the potential as a function of $\ell$ for $F(y)=y^{n}$, for various values of $.424 \leq n \leq 2$ and of $x=\left(m^{\prime}\right)^{\frac{1}{3}} q$ with $0 \leq x<1$, as required by positivity of the scalar metric. We found that, if the potential is bounded, the global minimum is always at $\langle y\rangle=\langle V\rangle=\langle W\rangle=0$, so supersymmetry is unbroken if the potential is bounded.

II. $b_{1}=0, b_{2}=b$.

This incorporates the Yang-Mills wave function renormalization at the condensation scale into the Kähler potential for $H$, but leaves the dilaton Kähler potential unmodified from its form at the string scale. A self-consistent physical interpretation requires strong coupling: $g_{c}^{2} \gg 1$ at the nonperturbative vacuum, where

$$
2 g_{c}^{2}=\ell_{c}=\frac{\ell}{1+3 b \ell \ln q}>\ell
$$


It turns out that that positivity of the kinetic energy together with the condition $\ell_{c}>\ell$ requires $\ell_{c} \leq 7.8$. Moreover, if we start in a region of parameter space where both the potential and the eigenvalues of the Kähler metric are positive, the potential goes to $+\infty$ as we approach the limiting value of $\ell_{c}$, which therefore cannot be reached. Alternatively, the potential is negative for positive eigenvalues of the Kähler metric, and goes to $-\infty$ in the limit. We conclude that this parameterization is inconsistent with condensation if the potential is bounded from below.

III. No scale case: $b_{1}=b_{2}=b$.

This corresponds to replacing the string coupling constant everywhere by the running coupling constant evaluated at the condensation scale, and results in a potential that is automatically positive semi-definite, because it satisfies the general criterion [18 for a no-scale potential. In this case the potential is minimized for $W_{0}=0, c=W$, so there is no supersymmetry breaking if $c=0$. If we take $F(y)=\alpha y^{n} \ln (y / \mu)$, we obtain $\left\langle W_{0}\right\rangle=0$ for $y=0$ or $y=\mu$. The interesting case is the latter one $(y \neq 0)$, for which the positivity constraints on the scalar metric are satisfied provided

$$
\mu>1, \quad \ell_{c}=\frac{\ell}{1+3 b \ell \ln q}=\frac{1}{6 b \ln \mu}>\frac{1}{b}=\frac{1}{.37} .
$$

Assuming $\alpha, \mu \sim 1$, and $\ell=g^{2} / 2=1 / 4$, the gravitino squared mass is given by

$$
m_{\tilde{G}}^{2}=\frac{\ell c^{2} e^{G}}{(1-x)^{3}}=\frac{\alpha^{2} \mu^{2 n} e^{-1 / b \ell_{0}}}{9 b^{2} \ell(1-x)^{3}} \sim \frac{\left(5 \times 10^{-4} m_{P l}\right)^{2}}{\alpha_{c} / 4 \pi},
$$

and the compactification radius is

$$
\Lambda_{\text {comp }}=(\operatorname{Ret} \operatorname{Re} s)^{-1} \sim .16 m_{P l} /\left(\alpha_{c} / 4 \pi\right)^{\frac{1}{6}}
$$

where $\alpha_{c}=\ell_{c} / 2 \pi$ is the fine structure constant at the condensation scale; consistency requires $\alpha_{c} / 4 \pi \geq O(1)$. 
A pertinent question is whether the use of $S$-duality constraints brings any qualitatively new features to the problem of gaugino condensation. We have not found a satisfactory supersymmetry breaking potential without introducing a constant $c$, which is the same as the situation without $S$-duality. A similar result was found in [10] where a different definition of $S$-duality was used. Moreover, if we take $M^{\prime} \rightarrow 1$ in (3.6) we find positivity constraints similar to those in III; taking $n=3$ in the superpotential of III, we recover the model studied in [15, 21, except that the Kähler potential has been corrected to include the Green-Schwarz anomaly cancellation counterterm, with the string coupling renormalized at the condensation scale $\mu\left(b_{1}=b\right)$ so as to recover a no-scale effective Lagrangian. Thus the implementation of approximate invariance under $S$-duality does not seem to qualitatively change the picture of gaugino condensation, at least in the chiral multiplet formulation.

\section{B. Linear multiplet formulation}

There is reason to believe that the linear multiplet formulation is the correct one for describing the dilaton supermultiplet from string theory, and, in fact, the Green-Schwarz counterterm is most easily constructed within this framework [5, 27]. While this formalism is dual to the chiral formalism in the tree approximation, it has generally been assumed that this duality may be broken by nonperturbative quantum effects. If this were true, one could interpret the potentials parameterized in the previous subsection in the following way. In the absence of a superpotential, the theory defined by the Kähler potential (3.6) is dual to a theory containing a linear supermultiplet $L$ and the chiral supermultiplets $T, H$ with Kähler potential $K=k(L, Q)+$ $G$, where $k(L, Q)$ is modular invariant. The Green-Schwarz counterterm appears 27] as a subtraction constant $V_{G S}$ in the integral equation

$$
S+\bar{S}+V_{G S}(G, Q)=-\int \frac{d L}{L} \frac{\partial K}{\partial L}
$$


obtained from integrating the equation of motion for $L$. One can choose the functions $V_{G S}$ and $k$ such that the Kähler potential (3.6) is recovered in the dual formulation in terms of the chiral supermultiplet $S$. Within this perspective, one would first perform the duality transformation to cast the theory in terms of a chiral supermultiplet, and then add a potential induced by nonperturbative quantum effects which should vanish in the limit of vanishing gauge coupling constant. This last requirement coincides with the $S$-duality constraint imposed in Section 3.A.

However, recent investigations [28, 29] suggest that chiral/linear multiplet duality may not be broken by nonperturbative effects, and that, in any case, gaugino condensation and the generation of a potential for the dilaton supermultiplet can be implemented directly within the linear supermultiplet formulation [28, 29, 26]. The physical degrees of freedom of the chiral supermultiplet are the dilaton $\sigma$ and and axion $a$. In the classical approximation to effective supergravity theories derived from string theory, the axion is dual to a three-form $h_{\mu \nu \rho}$ that is the curl of a two-form potential $b_{\mu \nu}$. The conventional wisdom has been that duality is preserved in the absence of a potential for the axion. However, it was shown some time ago [30] that interactions for the two-form can be introduced in such a way that the dual theory contains massive scalars. In the remainder of this subsection we will consider what the implications of $S$-duality may be in the general framework of gaugino condensation as formulated in terms of a linear supermultiplet. We will see that some of the difficulties encountered in the chiral multiplet formulation are avoided.

The only subgroup of $S L(2, \mathcal{R})$ defined by (2.14) and (2.19) that does not mix the dilaton $\sigma$ with the axion $a$ is the group of scale transformations:

$$
a^{-1}=d=\lambda, \quad b=c=0, \quad\left(S^{\prime}+\bar{S}^{\prime}\right)=\lambda^{-2}(S+\bar{S}), \quad W_{\alpha}^{\prime} \rightarrow \lambda W_{\alpha} .
$$

The transformation law for the linear multiplet $L$ can be inferred from (3.16),

\footnotetext{
${ }^{6}$ The symmetries involving the axion reemerge in the linear formalism as two independent gauge transformations of the transverse antisymmetric tensor $b_{\mu \nu}$.
} 
which gives, in the classical limit:

$$
L=(S+\bar{S})^{-1}, \quad L \rightarrow L^{\prime}=\lambda^{2} L
$$

This symmetry is respected by the modified linearity condition [27]

$$
\left(\mathcal{D}_{\dot{\alpha}} \mathcal{D}^{\dot{\alpha}}-8 R\right) L+k W^{\alpha} W_{\alpha}=0,
$$

where $k$ is a constant, when the Yang-Mills sector is included.

In references [29, 26] a vector supermultiplet $V$ was introduced that has among its components the components of a linear supermultiplet $L$ and of a chiral multiplet,

$$
U=-\left(\mathcal{D}_{\dot{\alpha}} \mathcal{D}^{\dot{\alpha}}-8 R\right) V
$$

that has the same Kähler weight as $W^{\alpha} W_{\alpha}$, and moreover satisfies the condition:

$$
\left(\mathcal{D}^{\alpha} \mathcal{D}_{\alpha}-24 R^{\dagger}\right) U-\left(\mathcal{D}_{\dot{\alpha}} \mathcal{D}^{\dot{\alpha}}-24 R\right) \bar{U}=\frac{i}{3} \epsilon_{m n p q} \partial^{m} \Gamma^{n p q},
$$

where $\Gamma_{A B C}$ is a super three-form gauge potential [31]. Eq. (3.21) is consistent with the constraint (3.2) if we interpret $U \sim W^{\alpha} W_{\alpha}$ as the condensate chiral multiplet.

In contrast to the case discussed in section 3.A, we do not need to introduce a "classical" superpotential for the composite supermultiplet $U$, because the corresponding term is implicitly included in the kinetic energy term for $V$ just as, in the linear multiplet formalism for the dilaton coupled to YangMills fields, the Yang-Mills lagrangian is implicitly included in the lagrangian for $L$ through the linearity condition (3.19).

In the case of global supersymmetry [29, 26] the "quantum" superpotential is the same as in (3.1):

$$
W(U)=\frac{b}{4} U \ln U
$$

If no additional operators are introduced in the lagrangian, the complex scalar field $u$ is a nonpropagating auxiliary field, but a potential is induced 
for the dilaton supermultiplet. The field $u$ can acquire a kinetic energy term with the introduction of appropriate operators of higher dimension. This construction was extended to the supergravity case in [26] using the formalism of superconformal supergravity, with $N=1$ Poincaré gauge fixing constraints imposed on the chiral compensator [32]. Here we use the Kähler covariant formulation [14], where $S$-duality, as defined by (3.18), is more transparent. In this case the classical kinetic energy term is just

$$
\mathcal{L}_{C}=-3 \int d^{4} \theta E\left[\alpha+\frac{1}{3} V V_{G S}\right],
$$

where $\alpha$ is a constant, and $V_{G S}=-b G$ is the Green-Schwarz counterterm introduced in (3.16). The nonperturbatively induced superpotential term is

$$
\mathcal{L}_{Q}=\frac{b}{8} \int d^{4} \theta \frac{E}{R} U \ln \left(e^{-K / 2} U\right)+\text { h.c. }, \quad K=\ln V+G,
$$

where the argument of the log, which must be a superfield of Kähler chiral weight $w=0$, can be understood [21] in terms of the ratio of the infrared cut-off $\left(U^{\frac{1}{3}}\right)$ and the effective ultraviolet cut-off $\left(e^{K / 6}\right)$. Under the modular transformation (3.5), with

$$
V^{\prime}(\theta)=V\left(\theta^{\prime}\right), \quad U^{\prime}(\theta)=e^{\frac{1}{2}(\bar{F}-F)} U\left(\theta^{\prime}\right),
$$

we have

$$
\delta \mathcal{L}_{C}=-\delta \mathcal{L}_{Q}=b \int d^{4} \theta E V(F+\bar{F})=\frac{b}{8} \int d^{4} \theta \frac{E}{R} U F+\text { h.c. } .
$$

If the kinetic term for the condensate is dominated by field theory quantum corrections and/or string corrections analogous to (3.10), that arise only from the dilaton/Yang-Mills/gravity sector interactions, then $S$-duality can be used as a guide to their construction. For example, in the general formalism described in [14], terms in the locally supersymmetric lagrangian are of the generic form

$$
\mathcal{L}=\frac{1}{2} \int \frac{E}{R} \Phi+\text { h.c. }
$$


where $\Phi$ is a chiral supermultiplet $\left(\mathcal{D}^{\dot{\alpha}} \Phi=0\right)$ with Kähler chiral weight $w(\Phi)=+2$. A special case is

$$
\mathcal{L}=\int E \phi, \quad w(\phi)=0, \quad \Phi=-\frac{1}{8}\left(\mathcal{D}_{\dot{\alpha}} \mathcal{D}^{\dot{\alpha}}-8 R\right) \phi .
$$

Superfields $\Phi, \phi$ can be constructed from $S$-duality invariant forms such as

$$
V^{-n} \mathcal{D}^{m} V^{n}, \quad \mathcal{D}^{m} \ln V, \quad \mathcal{D}=\mathcal{D}^{\alpha}, \mathcal{D}_{\dot{\alpha}}
$$

Terms similar to these were used in [26] to generate effective lagrangians for a dynamical condensate. The restricted class of models studied (which do not include a constant $c$ in the superpotential) do not break supersymmetry at the vacuum.

\section{Conclusions}

We have explicated the gauge and dilaton superfield transformations under the $S L(2, \mathcal{R})$ group of duality rotations that includes weak/strong coupling duality, $S \rightarrow 1 / S$, as a group element. $S L(2, \mathcal{R})$ is a symmetry of the Yang-Mills/dilaton/gravity sector in effective Lagrangians obtained from superstring theory, and we have studied its implications for models with a dynamical condensate. In the linear multiplet formulation for the dilaton, the only remnant of $S L(2, \mathcal{R})$ is a scale transformation that can nevertheless be used to constrain the operators appearing in the lagrangian, and in fact this formulation appears to be the more natural framework for describing a composite superfield condensate. None of the models studied so far have produced a bounded potential with supersymmetry breaking at the vacuum without the introduction of a constant term in the superpotential.

Acknowledgements. We thank Bruno Zumino for discussions, and Kamran Saririan and Yi-Yen Wu for a careful reading of the manuscript. This work was supported in part by the Director, Office of Energy Research, Office of 
High Energy and Nuclear Physics, Division of High Energy Physics of the U.S. Department of Energy under Contract DE-AC03-76SF00098 and in part by the National Science Foundation under grant PHY-90-21139.

\section{References}

[1] M.K. Gaillard and B. Zumino, Nucl. Phys. B193: 221 (1981).

[2] S. Ferrara, J. Scherk and B. Zumino, Nucl. Phys. B121: 393 (1977).

[3] A. Giveon, N. Malkin and E. Rabinovici, Phys. Lett. B220: 551 (1989);

E. Alvarez and M. Osorio, Phys. Rev. D40: 1150 (1989).

[4] L. Dixon, V. Kaplunovsky and J. Louis, Nucl. Phys. B355: 649 (1991).

[5] G.L. Cardoso and B.A. Ovrut, Nucl. Phys. B369: 351 (1992); J.-P. Derendinger, S. Ferrara, C. Kounnas and F. Zwirner, Nucl. Phys. B372: 145 (1992).

[6] A. Font, L. Ibáñez, D. Lüst and F. Quevedo, Phys Lett. 249B: 35 (1990).

[7] J.H. Schwarz and A. Sen, Nucl. Phys. B411: 35 (1994).

[8] J.H. Schwarz and A. Sen, Phys. Lett. B312: 105 (1993); J.H. Schwarz, CALT-68-1965 hep-th/9411178 (1994); M. Duff, NI-94-033/hepth/9501030 (1994); E. Witten, IASSNS-HEP-95-18/hep-th/9503124 (1995).

[9] A. Sen, Int. J. Mod. Phys. A9 3707 (1994); N. Seiberg and E. Witten, Nucl. Phys. B431: 484 (1994).

[10] Z. Lalak, A. Niemeyer and H.P. Nilles, Phys. Lett. 349B: 99 (1995), and TUM-HEP-211/1995, hep-th/9503170.

[11] G. Veneziano and S. Yankielowicz, Phys. Lett. 113B: 231 (1982). 
[12] T.R. Taylor, Phys. Lett. 164B: 43 (1985).

[13] E. Cremmer, S. Ferrara, L. Girardello, and A. Van Proeyen, Nucl. Phys. B212: 413 (1983).

[14] P. Binétruy, G. Girardi, R. Grimm and M. Muller, Phys. Lett. 189B: 83 (1987); P. Binétruy, G. Girardi and R. Grimm, Annecy preprint LAPPTH-275-90, (1990).

[15] P. Binétruy and M.K. Gaillard, Phys. Lett. 232B: 82 (1989).

[16] S. Deser and C. Teitelboim, Phys. Rev. D13: 1592 (1976).

[17] S. Ferrara, N. Magnoli, T.R. Taylor and G. Veneziano, Phys. Lett. B245 409 (1990).

[18] P. Binétruy and M.K. Gaillard, Phys. Lett. 253B: 119 (1991).

[19] A. de la Macorra and G.G. Ross, Nucl. Phys. B404 321 (1993); E. Dudas, Ph. D. thesis, Orsay University (1994); R. Peschanski and C.A. Savoy, preprint SACLAY-SPHT-95-002/hep-ph/9504243 (1995).

[20] I. Antoniadis, E. Gava, K.S. Narain and T.R. Taylor, Nucl. Phys. B432: 187 (1994).

[21] P. Binétruy and M.K. Gaillard, Nucl. Phys. B358: 121 (1991).

[22] M.K. Gaillard and T.R. Taylor, Nucl. Phys. B381: 577 (1992).

[23] P. Mayr and S. Stieberger, Nucl. Phys. B412: 502 (1994); V. Kaplunovsky and J. Louis, preprint UTTG-24-94/LMU-TPW-94-24 (1995).

[24] M. Dine, R. Rohm, N. Seiberg and E. Witten, Phys. Lett. 156B: 55 (1985). 
[25] M.K. Gaillard, V. Jain and K. Saririan, LBL-34948, preprint in preparation.

[26] P. Binétruy, M.K. Gaillard, and T.R. Taylor, preprint LBL-36744/UCB95/03/NUB-3117/LPTHE-Orsay 95/14/hep-th/9504143 (1995).

[27] P. Binétruy, G. Girardi, R. Grimm and M. Müller, Phys. Lett. B265 111 (1991); P. Adamietz, P. Binétruy, G. Girardi and R. Grimm, Nucl. Phys. B401: 257 (1993).

[28] I. Gaida and D. Lüst, preprint HUB-IEP-94/33/hep-th/9412079.

[29] C.P. Burgess, J.P. Derendinger, F. Quevedo and M. Quirós, Phys. Lett. B348: 428 (1995).

[30] A. Aurilia, Y. Takahashi and P.K. Townsend, Phys. Lett. 95: 265 (1980); A. Aurilia and Y. Takahashi, Prog. of Theor. Phys. 66: 693 (1981).

[31] S.J. Gates, Nucl. Phys. B184: 381 (1981); G. Girardi and R. Grimm, Phys. Lett. B260: 365 (1991); P. Binétruy, G. Girardi, R. Grimm and F. Pillon, paper in preparation.

[32] T. Kugo and S. Uehara, Nucl. Phys. B222 125 (1983); Nucl. Phys. B226 49 (1983). 